Supporting information for:

\title{
Direct analysis of ion-induced peptide fragmentation in secondary ion mass spectrometry
}

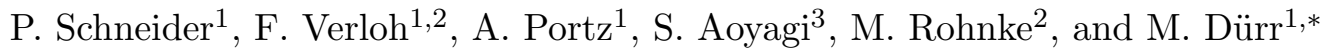 \\ ${ }^{1}$ Institut für Angewandte Physik and Zentrum für Materialforschung, Justus-Liebig-Universität \\ Giessen, Heinrich-Buff-Ring 16, D-35392 Giessen, Germany \\ ${ }^{2}$ Physikalisch-Chemisches Institut and Zentrum für Materialforschung, Justus-Liebig-Universität \\ Giessen, Heinrich-Buff-Ring 17, D-35392 Giessen, Germany \\ ${ }^{3}$ Seikei University, Department of Materials and Life Science, 3-3-1, Kichijyoji-kitamachi, \\ Musashino-shi, Tokyo 180-8633, Japan \\ *Corresponding author: michael.duerr@ap.physik.uni-giessen.de
}

This supporting information includes

(I) Experimental parameters for the TOF-SIMS measurements

(II) List of fragment peaks observed in DINeC and TOF-SIMS spectra

(III) Mass spectra on logarithmic scale

(IV) Description of the analytical model covering the dependence of fragmentation on depth and fluence

(V) Influence of the functional form of $f(d)$ on calculated DINeC and SIMS signals

(VI) Parameter values used to fit the analytical model to the experimental data

(VII) Calculated DINeC and SIMS signals in the high-fluence limit 


\section{Experimental parameters for the TOF-SIMS measurements}

Table S1 summarizes the experimental parameters applied in the TOF-SIMS measurements.

TABLE S1: Experimental parameters for long time ion exposure during TOF-SIMS measurements

\begin{tabular}{|l|l|l|l|l|l|l|}
\hline Species & Energy & Current & $\begin{array}{l}\text { Cycle } \\
\text { time }\end{array}$ & $\begin{array}{l}\text { Pixel } \\
\text { number }\end{array}$ & $\begin{array}{l}\text { Shots per } \\
\text { pixel }\end{array}$ & $\begin{array}{l}\text { Mass resolution } \\
\text { FWHM } \\
m / \Delta m @ m / z \\
1046.5\end{array}$ \\
\hline $\mathrm{Bi}^{+}$ & $25 \mathrm{keV}$ & $0.78 \mathrm{pA}$ & $140 \mu \mathrm{s}$ & $128 \times 128$ & 1 & $>4300$ \\
$\mathrm{Bi}_{3}^{+}$ & $25 \mathrm{keV}$ & $0.25-0.28 \mathrm{pA}$ & $110 \mu \mathrm{s}$ & $128 \times 128$ & 1 & $>8300$ \\
$\mathrm{Ar}_{1000}^{+}$ & $20 \mathrm{keV}$ & $0.11-0.13 \mathrm{pA}$ & $140 \mu \mathrm{s}$ & $128 \times 128$ & 1 & $>7100$ \\
\hline
\end{tabular}

For the combined SIMS-DINeC experiments, large scale irradiation of the whole angiotensin II dots was performed. For $\mathrm{Bi}^{+}$and $\mathrm{Bi}_{3}^{+}$irradiation, the $2 \mathrm{D}$ large area mode (stage scan) was applied and the sample was scanned with the ion beam until the appropriate fluence was achieved. In any case, complete cycles were carried out to obtain a homogeneous fluence over the whole sample area. Due to the low current of the argon ion gun in analysis mode, the gun was operated as sputter gun for the large area irradiation experiments. Here we increased the delay parameter of the ion gun pulser to $56 \mu$ s in order to reduce the current to about $2 \mathrm{nA}$. For the measurements, the ion beam scanned an area of $700 \times 700 \mu \mathrm{m}^{2}$. For $\mathrm{Ar}_{1000}^{+}$primary ions, the large scan 2D measurement mode cannot be used within the IONTOF surface lab software. Thus, we implemented the following procedure in order to perform the $\mathrm{Ar}_{1000}^{+}$irradiation: we moved the stage in meander mode during scanning, then started the secondary ion acquisition, deactivated the primary ion gun, and switched on the sputter gun manually. Stage moving speeds were in the range between 0.05 and $3.00 \mathrm{~mm} / \mathrm{s}$ depending on the required fluence. The cluster size distribution of argon primary cluster ions used in this type of experiment is depicted in Fig. S1. 


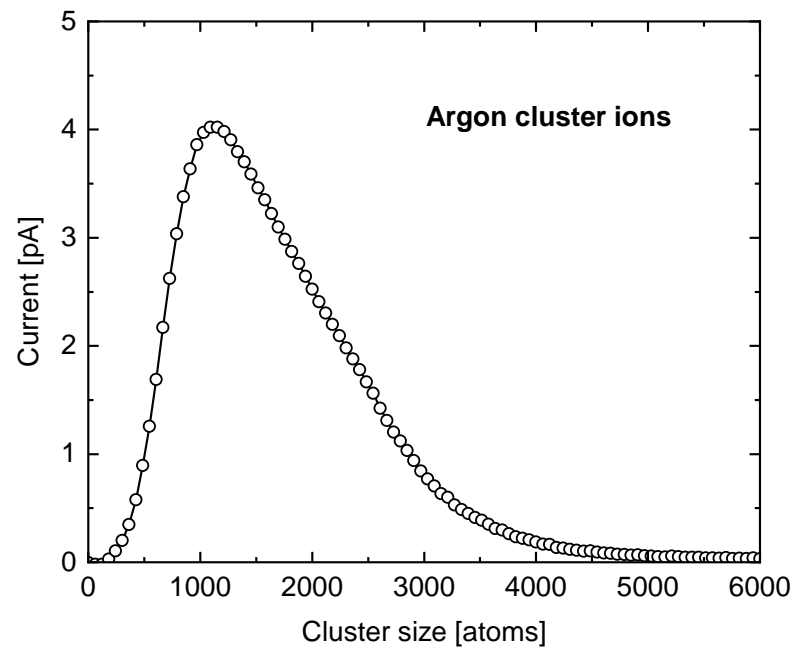

FIG. S1: The cluster size distribution for $\mathrm{Ar}_{1000}^{+}$primary ions used in the irradiation experiments. 


\section{List of fragment peaks observed in DINeC and TOF-SIMS spectra}

Table S2 contains a list of the most pronounced peaks observed in the TOF-SIMS and the DINeC spectra. Molecular formulas of identified fragments are also included. For specific fragments, the fragment denotation is displayed along with the $m / z$ values and the molecular formula.

TABLE S2: Fragment ion peaks observed in the mass spectra obtained by means of TOF-SIMS and DINeC.

\begin{tabular}{|c|c|c|c|c|}
\hline$m / z$ & denotation & molecular formula & $\begin{array}{r}\text { pres } \\
\text { TOF-SIMS } \\
\end{array}$ & $\begin{array}{l}\text { ent in } \\
\text { DINeC }\end{array}$ \\
\hline 30.0 & - & $\mathrm{CH}_{4} \mathrm{~N}$ & $\mathrm{x}$ & - \\
\hline 59.0 & - & $\mathrm{CH}_{5} \mathrm{~N}_{3}$ & $\mathrm{x}$ & $\mathrm{x}$ \\
\hline 70.1 & - & $\mathrm{C}_{4} \mathrm{H}_{8} \mathrm{~N}$ & $\mathrm{x}$ & - \\
\hline 73.1 & - & $\mathrm{C}_{2} \mathrm{H}_{7} \mathrm{~N}_{3}$ & $\mathrm{x}$ & $\mathrm{x}$ \\
\hline 81.0 & - & $\mathrm{C}_{4} \mathrm{H}_{5} \mathrm{~N}_{2}$ & $\mathrm{x}$ & - \\
\hline 100.1 & - & $\mathrm{C}_{4} \mathrm{H}_{10} \mathrm{~N}_{3}$ & $\mathrm{x}$ & - \\
\hline 107.0 & - & $\mathrm{C}_{7} \mathrm{H}_{7} \mathrm{O}$ & $\mathrm{x}$ & - \\
\hline 110.1 & - & $\mathrm{C}_{5} \mathrm{H}_{8} \mathrm{~N}_{3}$ & $\mathrm{x}$ & - \\
\hline 136.1 & - & $\mathrm{C}_{8} \mathrm{H}_{10} \mathrm{NO}$ & $\mathrm{x}$ & - \\
\hline 207.0 & - & - & $\mathrm{x}$ & - \\
\hline 221.1 & - & - & $\mathrm{x}$ & $\mathrm{x}$ \\
\hline 249.0 & - & - & $\mathrm{x}$ & - \\
\hline 265.0 & - & - & $\mathrm{x}$ & $\mathrm{x}$ \\
\hline 281.0 & - & - & $\mathrm{x}$ & - \\
\hline 289.0 & $\mathrm{c}_{2} / \mathrm{x}_{2}$ & $\mathrm{C}_{10} \mathrm{H}_{21} \mathrm{~N}_{6} \mathrm{O}_{4} / \mathrm{C}_{15} \mathrm{H}_{17} \mathrm{~N}_{2} \mathrm{O}_{4}$ & $\mathrm{x}$ & $\mathrm{x}$ \\
\hline 295.0 & - & - & $\mathrm{x}$ & - \\
\hline 309.0 & - & - & $\mathrm{x}$ & - \\
\hline 325.0 & - & - & $\mathrm{x}$ & - \\
\hline 343.3 & $a_{3}$ & $\mathrm{C}_{14} \mathrm{H}_{27} \mathrm{~N}_{6} \mathrm{O}_{4}$ & $\mathrm{x}$ & - \\
\hline 355.1 & - & - & $\mathrm{x}$ & - \\
\hline 385.0 & - & - & $\mathrm{x}$ & - \\
\hline 403.3 & - & - & $\mathrm{x}$ & $\mathrm{x}$ \\
\hline 413.3 & - & - & $\mathrm{x}$ & $\mathrm{x}$ \\
\hline 425.3 & - & - & $\mathrm{x}$ & $\mathrm{x}$ \\
\hline 441.3 & - & - & $\mathrm{x}$ & $\mathrm{x}$ \\
\hline 469.3 & - & - & - & $\mathrm{x}$ \\
\hline 485.3 & - & - & - & $\mathrm{x}$ \\
\hline 506.3 & $\mathrm{a}_{4}$ & $\mathrm{C}_{23} \mathrm{H}_{36} \mathrm{~N}_{7} \mathrm{O}_{6}$ & $\mathrm{x}$ & - \\
\hline 551.3 & $\mathrm{c}_{4}$ & $\mathrm{C}_{24} \mathrm{H}_{39} \mathrm{~N}_{8} \mathrm{O}_{7}$ & $\mathrm{x}$ & - \\
\hline 556.3 & - & - & - & $\mathrm{x}$ \\
\hline 591.3 & - & - & $\mathrm{x}$ & - \\
\hline
\end{tabular}




\begin{tabular}{|c|c|c|c|c|}
\hline$m / z$ & denotation & molecular formula & $\begin{array}{r}\text { pres } \\
\text { TOF-SIMS }\end{array}$ & $\begin{array}{l}\text { ent in } \\
\text { DINeC }\end{array}$ \\
\hline 619.4 & $a_{5}$ & $\mathrm{C}_{29} \mathrm{H}_{47} \mathrm{~N}_{8} \mathrm{O}_{7}$ & $\mathrm{x}$ & - \\
\hline 664.4 & $c_{5}$ & $\mathrm{C}_{30} \mathrm{H}_{50} \mathrm{~N}_{9} \mathrm{O}_{8}$ & $\mathrm{x}$ & $\mathrm{x}$ \\
\hline 756.5 & $a_{6}$ & $\mathrm{C}_{35} \mathrm{H}_{54} \mathrm{~N}_{11} \mathrm{O}_{8}$ & $\mathrm{x}$ & - \\
\hline 786.5 & $\mathrm{~b}_{6}+2 \mathrm{H}$ & $\mathrm{C}_{36} \mathrm{H}_{56} \mathrm{~N}_{11} \mathrm{O}_{9}$ & - & $\mathrm{x}$ \\
\hline 875.2 & - & - & - & $\mathrm{x}$ \\
\hline 898.4 & $c_{7}$ & $\mathrm{C}_{41} \mathrm{H}_{64} \mathrm{~N}_{13} \mathrm{O}_{10}$ & $\mathrm{x}$ & $\mathrm{x}$ \\
\hline 916.4 & $\mathrm{z}_{7}+2 \mathrm{H}$ & $\mathrm{C}_{46} \mathrm{H}_{66} \mathrm{~N}_{11} \mathrm{O}_{9}$ & $\mathrm{x}$ & $\mathrm{x}$ \\
\hline 1002.5 & $\mathrm{a}_{8}+2 \mathrm{H}$ & $\mathrm{C}_{49} \mathrm{H}_{72} \mathrm{~N}_{13} \mathrm{O}_{10}$ & $\mathrm{x}$ & $\mathrm{x}$ \\
\hline 1031.5 & $\mathrm{z}_{8}+2 \mathrm{H}$ & $\mathrm{C}_{50} \mathrm{H}_{71} \mathrm{~N}_{12} \mathrm{O}_{12}$ & $\mathrm{x}$ & $\mathrm{x}$ \\
\hline 1046.5 & {$[\mathrm{M}+\mathrm{H}]$} & $\mathrm{C}_{50} \mathrm{H}_{72} \mathrm{~N}_{13} \mathrm{O}_{12}$ & $\mathrm{x}$ & $\mathrm{x}$ \\
\hline
\end{tabular}




\section{Mass spectra on logarithmic scale}

In Fig. S2, the TOF-SIMS spectrum shown in Fig. 2 of the main article is depicted with the intensity plotted both on linear and on logarithmic scale.

In Fig. S3, the mass spectra of the $m / z$ region around 400 are shown with the intensity plotted on logarithmic scale for both DINeC and SIMS measurement (see Fig. 3 in the main article for the linear representation of the spectra). Arrows in the SIMS spectrum denote peak groups corresponding to isolated peaks in the DINeC spectrum (see main article for detailed discussion). Whereas broader peak groups are observed in the SIMS spectrum, the logarithmic representation shows that in the DINeC spectrum the indicated peaks are not accompanied by neighboring peaks towards lower $m / z$ values next to the main peak.
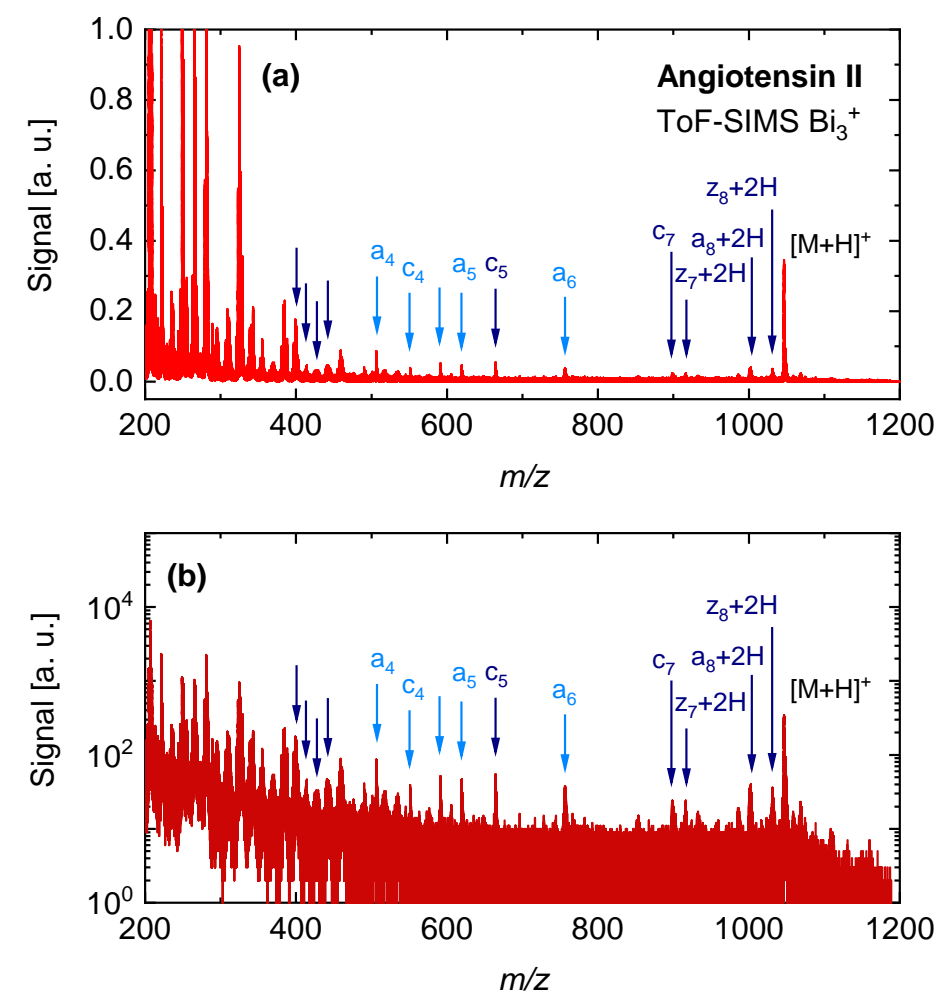

FIG. S2: TOF-SIMS spectrum (positive ion mode) from an ATII sample obtained during $\mathrm{Bi}_{3}^{+}$ bombardment (25 keV primary ion energy) on linear (a) and logarithmic scale (b). 

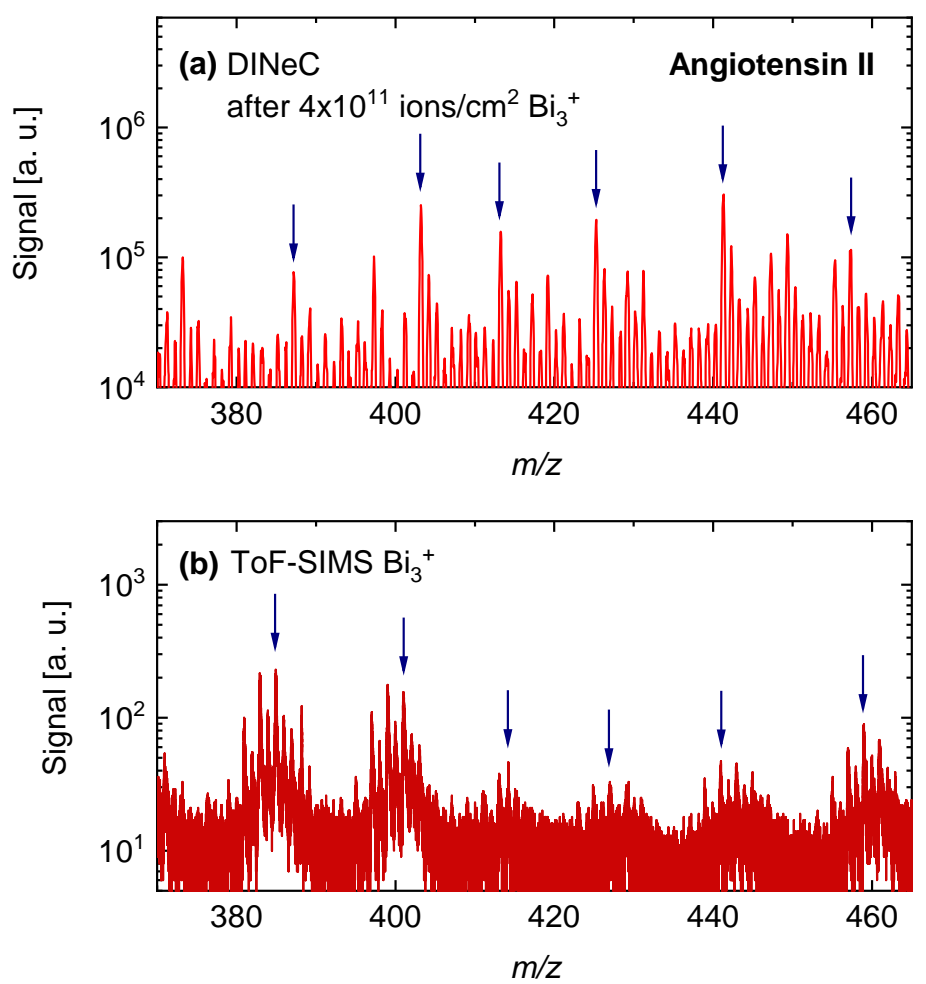

FIG. S3: Detailed view of the $m / z$ region around 400 in the DINeC (a) and the TOF-SIMS spectra (b) with intensity plotted on logarithmic scale. As in Fig. 3 in the main article, corresponding peak groups in the spectra are denoted by arrows. 


\section{Description of the analytical model covering the dependence of fragmenta- tion on depth and fluence}

In the model, we describe the fragmentation in the sample in terms of the relative concentration of intact molecules, $c$. In general, $c$ depends on the location within the sample and on the primary ion fluence $F$ the sample has been irradiated with. We assume that the primary ion beam covers the whole sample area homogeneously, so $c$ does not depend on the lateral position on the sample but only on the coordinate normal to the surface, $z$. Prior to irradiation, all molecules are assumed to be intact, thus $c(z, F=0)=1$.

Irradiation of the sample with primary ions changes the state of the sample in two ways. First, primary ion bombardment leads to sputtering of sample material and thus, the location of the sample surface changes. With the thickness of the sputtered sample material per primary ion fluence, $r$, the location of the surface for a given primary ion fluence is $z_{\mathrm{S}}(F)=r \cdot F$. It is important to note that $c(z, F)$ is only a reasonable parameter for $z \geq z_{\mathrm{S}}(F)$, i.e., for positions inside the sample.

Second, some of the molecules in the sample are fragmented due to primary ion irradiation. We describe this in terms of a fragmentation strength $f(d)$ which depends on the depth in the sample, $d$. It is defined such that for a small fluence $\mathrm{d} F$, the probability for fragmentation of an intact molecule in depth $d$ is given by $f(d) \mathrm{d} F$. The fragmentation strength vanishes for high values of $d$. Like $z$, the depth coordinate $d$ is directed perpendicular to the surface, however it is defined relative to the current $z$-position of the sample surface. Both the fragmentation strength $f$ and the sputtered depth per fluence $r$ depend on the primary ion species as well as on the composition of the sample.

To calculate the fragmentation induced by a small fluence $\mathrm{d} F$, the concentration of intact molecules is multiplied with the fragmentation strength relative to the current sample surface. This leads to the differential equation

$$
\frac{\mathrm{d} c}{\mathrm{~d} F}(z, F)=-f\left(z-z_{\mathrm{S}}(F)\right) c(z, F)
$$

This differential equation is solved by

$$
c(z, F)=\exp \left[-\int_{0}^{F} f\left(z-z_{\mathrm{S}}\left(F^{\prime}\right)\right) \mathrm{d} F^{\prime}\right]
$$

Finally, $c(z, F)$ can be used to model the DINeC and the SIMS signals based on a given functional form of the fragmentation strength $f(d)$. The DINeC signal is assumed to reflect 
only the concentration on the surface of the sample. Therefore, it is given by

$$
c_{\mathrm{DINeC}}(F)=c\left(z_{\mathrm{S}}(F), F\right)=\exp \left[-\int_{0}^{F} f\left(z_{\mathrm{S}}(F)-z_{\mathrm{S}}\left(F^{\prime}\right)\right) \mathrm{d} F^{\prime}\right]
$$

The SIMS signal reflects a mean concentration within a near-surface sample region. The thickness of this region can be expressed in terms of an information depth $d_{\mathrm{I}}$. The SIMS signal is then given by

$$
c_{\mathrm{SIMS}}(F)=\frac{1}{d_{\mathrm{I}}} \int_{z_{\mathrm{S}}(F)}^{z_{\mathrm{S}}(F)+d_{\mathrm{I}}} c(z, F) \mathrm{d} z
$$

To apply this model on an actual measurement, it is necessary to parameterize $f(d)$ based on a fixed functional relation and use the parameters to fit the model to the experimental data. Since different functional forms of $f(d)$ can lead to similar results for the signal intensity, the underlying fragmentation strength is not necessarily accurate, even though calculation and experiment match well. Besides, the model is based on assumptions which generally do not hold in real experiments, such as the constant value of the sputter depth $r$. Nevertheless, the model allows valuable insight into primary-ion-induced fragmentation when comparing different measurements, as long as the calculations are based on the same functional form for the fragmentation strength. In particular, the model can be applied to characterize important properties, such as the information depth and the fragmentation depth for different primary ions. 


\section{Influence of the functional form of $f(d)$ on calculated DINeC and SIMS signals}

In order to understand how calculated SIMS and DINeC signals depend on the functional form of the fragmentation strength, we used in a first approach a step function $f_{\text {step }}(d)=C_{\mathrm{F}} \theta\left(d_{\mathrm{F}}-d\right)$ as a simple function to parameterize the fragmentation strength, introducing a fragmentation strength parameter $C_{\mathrm{F}}$ and a fragmentation depth parameter $d_{\mathrm{F}}$. For this function, the DINeC and SIMS signals can be calculated analytically using Eqs. 3 and 4 . The dependence of both signals on fluence then follows an exponential decay function $\propto \exp \left(-C_{\mathrm{F}} F\right)$ in the low fluence regime and a constant contribution for higher fluences.

Fig. S4 shows the experimental data for samples irradiated with $\mathrm{Ar}_{1000}^{+}$clusters together with model calculations based on such a step function for the fragmentation strength. The figure clearly shows that the SIMS signal can be described well, even with such a simple fragmentation strength function. However, it turns out that there is no parameter set that fits the DINeC data well at the same time. In fact, with the fragmentation strength based on a single step function, it is only possible to fit either the DINeC or the SIMS data well. Further calculations show, that the results obtained with a single exponential decay function

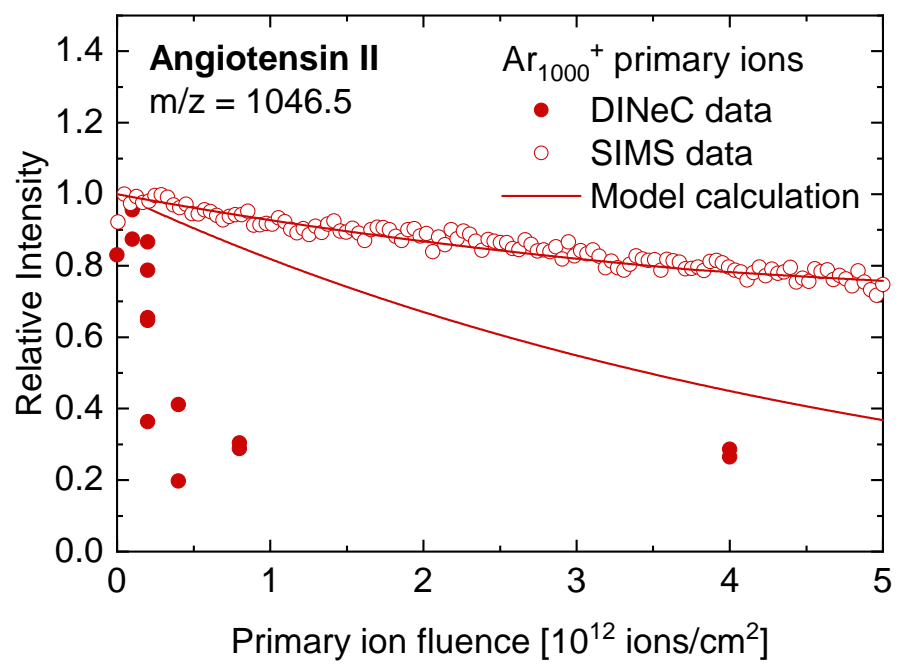

FIG. S4: Signal intensity corresponding to intact angiotensin II molecules measured in DINeC and SIMS experiments as a function of $\mathrm{Ar}_{1000}^{+}$primary ion dose. The lines represent model calculations of the SIMS and DINeC signal based on a single step function for fragmentation strength. The model parameters have been chosen in a way to give the best representation of the experimental data of the SIMS signal. At the same time, a good agreement of the calculated DINeC signal with the experimental data could not be achieved. 
instead of the step function show the same behavior. Therefore, the fragmentation strength function has to be extended in order to describe both signals.

We thus introduced functional forms of the fragmentation strength which consist of a longrange contribution and a short-range contribution. Calculations based on two exponential decay functions for the fragmentation strength are shown in Fig. 4 in the main article. To fit both signals, the short-range contribution to the fragmentation strength is typically much stronger than the long-range contribution. This high fragmentation close to the surface leads to the fast drop observed in the DINeC signal. Since the SIMS signal is also influenced by the sub-surface region, the strong fragmentation on the surface is less important and the signal drop is less pronounced. For comparison, the fitting results for the $\mathrm{Ar}_{1000}^{+}$ions when using $f(d)$ being composed of two step functions are shown in Fig. S5. The results are consistent with the results shown in Fig. 4 of the main article.

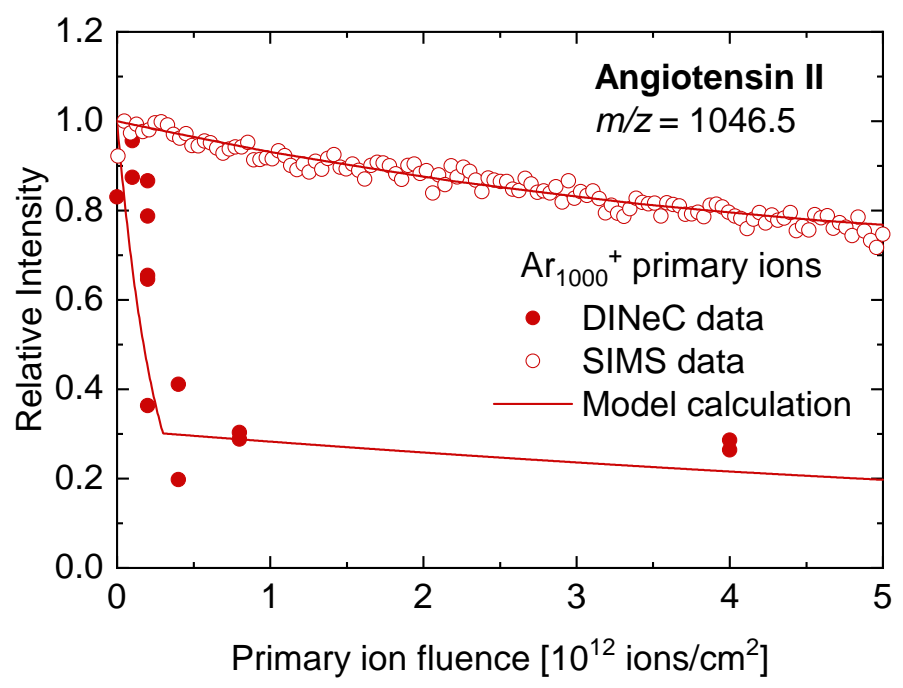

FIG. S5: Signal intensity corresponding to intact angiotensin II molecules desorbed in DINeC and SIMS experiments as a function of $\mathrm{Ar}_{1000}^{+}$primary ion dose. The lines represent model calculations of the SIMS and DINeC signal based on a fragmentation strength composed of two step functions. In the calculated DINeC signal, a kink is observed due to the discontinuity in the fragmentation strength. 
The parameters used to model the experimental data (compare Figs. 4 and 5 in the main article) are shown in table $\mathrm{S} 3$. The fragmentation depth parameters $d_{\mathrm{F}, 1}$ and $d_{\mathrm{F}, 2}$ as well as the corresponding fragmentation strength parameters $C_{1}$ and $C_{2}$ define the fragmentation strength as a function of depth $f(d)=C_{1} \cdot \exp \left(-d / d_{\mathrm{F}, 1}\right)+C_{2} \cdot \exp \left(-d / d_{\mathrm{F}, 2}\right)$.

TABLE S3: Model parameters used to fit the experimental data

\begin{tabular}{|l|l|l|l|l|}
\hline Species & $C_{1}\left[\mathrm{~nm}^{2}\right]$ & $C_{2}\left[\mathrm{~nm}^{2}\right]$ & $d_{\mathrm{F}, 1} / d_{\mathrm{I}}$ & $d_{\mathrm{F}, 2} / d_{\mathrm{I}}$ \\
\hline $\mathrm{Bi}^{+}$ & 1040 & 60 & 0.33 & 20 \\
$\mathrm{Bi}_{3}^{+}$ & 956 & 44 & 0.05 & 33 \\
$\mathrm{Ar}_{1000}^{+}$ & 438 & 12 & 0.025 & 0.83 \\
\hline
\end{tabular}




\section{Calculated DINeC and SIMS signals in the high-fluence limit}

Fig. S6 shows the calculated DINeC and SIMS signals based on the parameters used to fit the experimental data obtained with Ar cluster irradiation. Compared to Fig. 4 in the main article, Fig. S6 covers an extended fluence range. In particular, a steady state is reproduced by our model calculations (see main article for discussion).

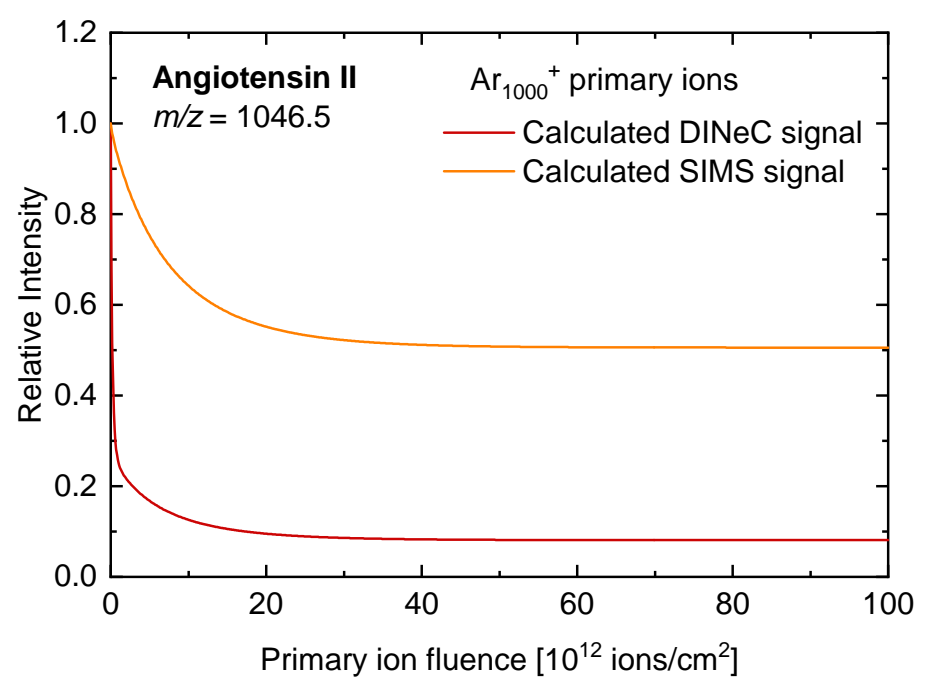

FIG. S6: Calculated DINeC and SIMS signals as shown in Fig. 4 in the main article, but for an extended fluence range. Towards higher fluence, the signal intensity reaches a steady state. 